\title{
A Seventeen-Year Descriptive Study of Infective Endocarditis Features at a Tertiary, Teaching Hospital
}

Saleh A. Alghamdi ${ }^{1}$, Abdulaziz M. Alkhammash ${ }^{1}$, Abdulrahman F. Alotaibi ${ }^{1}$, Saeed A. Bugshan ${ }^{1}$, Nawaf K. Alshanbri ${ }^{1}$, Mohammed Zahrani ${ }^{2}$

1. College of Medicine, King Abdulaziz University, Jeddah, SAU 2. Internal Medicine, King Abdulaziz University, Jeddah, SAU

Corresponding author: Saleh A. Alghamdi, i97ss@hotmail.com

\section{Abstract}

\section{Introduction}

Infective endocarditis (IE) is a microbial infection affecting the endothelial lining of the heart chambers and valves. Despite advances in diagnostic methods and management, IE still carries high levels of morbidity and mortality. There are no comprehensive data describing incidence, risk factors, and outcomes of IE in Saudi Arabia.

Our study aims to describe the epidemiological and clinical characteristics and outcomes of adult patients with IE treated in a tertiary, Teaching Hospital.

\section{Methods}

This is a descriptive, retrospective review of charts (between January 2003 and June 2019) conducted at King Abdulaziz University Hospital. We included all patients 16 years and older with a definitive diagnosis of IE based on Duke Criteria. We collected the following information: demographics, risk factors, comorbidities, microbial type, antibiotic choice, complications, laboratory data, echocardiography data, and mortality.

\section{Results}

We found a total of 60 adult patients with definitive diagnoses of IE: $55 \%$ of them were males, and the mean age was $48.71 \pm 18.3$ years. Hypertension was the most common comorbidity, affecting 23 patients (38.3\%), followed by end-stage renal disease (ESRD) in 20 patients (33.3\%) and diabetes in 17 (28.3\%). Staphylococcus aureus was the most common organism (25\%), and vancomycin was the most prescribed antibiotic. Fiftyeight patients were treated medically (96.5\%). Furthermore, $88 \%$ of the patients had vegetations, detected by echocardiography, and the majority had single-valve involvement. Sepsis was the most common complication, and the mortality rate was $36.7 \%$.

Review began 06/11/2021 Review ended 06/15/2021 Published 06/28/2021

\section{Copyright 2021}

Alghamdi et al. This is an open access article distributed under the terms of the Creative Commons Attribution License CC-BY 4.0., which permits unrestricted use, distribution, and reproduction in any medium, provided the original author and source are credited.

\section{Conclusion}

There was a small number of definitive IE cases over a 17-years span in our institution. Surprisingly, a higher mortality rate was found among our patients and a lower surgical intervention rate in comparison with the literature. Though we acknowledge the limitations of a retrospective, chart review study, we can speculate that the high mortality rate may be in part related to a higher number of virulent organisms, delayed presentation, and lack of prompt surgical intervention.

Categories: Cardiac/Thoracic/Vascular Surgery, Cardiology, Infectious Disease

Keywords: infective endocarditis, echocardiography, vegetation, modified duke criteria, sepsis, embolization

\section{Introduction}

Infective endocarditis (IE) is a life-threatening, multisystemic illness initiated by microbial infection, mostly bacterial, targeting the endothelial lining of the heart chambers and valves. It could also affect the mural endothelium, chordae tendineae, interventricular septum, and other intracardiac tissues or devices [1-3].

The modified Duke Criteria is the recommended diagnostic method to follow for diagnosing IE according to recent guidelines [4]. However, despite the huge advances in diagnostic methods as well as antimicrobial, medical, and surgical techniques of management nowadays, IE still shows grave levels of morbidity and mortality [5,6], with a five-year mortality rate exceeding 40\% [7]. IE could also result in lethal conditions such as embolic events (most seriously in the form of embolic stroke), disseminated infections throughout the body, congestive heart failure, and organ failure; and these complications are common. In addition, IE could deteriorate to DIC or lead to fatal multiorgan failure [8]. The incidence of at least one complication 
could affect as much as half the patients diagnosed with IE [9].

Clinical risk factors for IE include the following: advanced age (above 58 years), male gender, patients who have prolonged vascular access, intravenous drug use, implanted intracardiac devices, as well as prosthetic valves. Furthermore, several comorbidities have been correlated with increased risk of IE development, including chronic kidney disease (CKD), congenital heart diseases (CHD), cancer, human immunodeficiency virus (HIV), diabetes mellitus (DM), and previous dental procedures. Furthermore, contact with the healthcare system itself is considered a risk factor for developing IE [7,10-13].

An important predisposing factor is a chronic hemodialysis $(\mathrm{CH})$. Patients with $\mathrm{CKD}$ are more susceptible to infections and the mortality rate is high, where half of these patients will not survive [14,15]. CH patients have a higher incidence of IE compared with those receiving peritoneal dialysis or kidney transplantation [16].

In developing countries, IE continues to be related to rheumatic heart disease (RHD), while in developed countries, patients are older and referred to healthcare facilities, and degenerative or prosthetic valves, as well as cardiovascular implantable electronic devices, have replaced RHD as the trending risk factor [17,18]. A study has concluded that patients with simple congenital ventricular septal defects (VSDs) have up to a 15fold increased risk of IE compared with the general population, and those with unrepaired VSDs have a considerably higher risk [19].

Globally, the incidence of IE ranges from 1.5 to 11.6 cases per 100,000 persons per year, peaking at 15 patients per 100,000 of the population in the United States (US) in 2011. The male to female ratio is over $1.5: 1[1,2,20,21]$. The most commonly affected valve in IE patients is the mitral valve, followed by the aortic, tricuspid valve, and less frequently, the pulmonary valve [22].

According to a recent huge prospective study done in Turkey, Staphylococcus aureus is the most common causative organism behind IE, which has been found in more than a third of patients, followed by Streptococcus viridans, and Enterococci [23]. Another 10-year retrospective study, carried out in Tunisia, showed that rheumatic valve disease was noted in $45.2 \%$ of native valve IE. In the study, no instance of IE was related to IV medication use, and staphylococci were the most prevalent microorganisms (17.9\%), and congestive heart failure was the most common complication, occurring in $45.2 \%$ [24].

A local study in Saudi Arabia stated that native valves were more frequently affected by IE than prosthetic valves. In particular, the mitral valve was the most commonly affected valve. On the other hand, $31.4 \%$ of cases had undergone surgical management, with an in-hospital mortality rate of $29.4 \%$ [25].

There are not enough studies investigating IE in Saudi Arabia with good, representative sample sizes, and over long periods of time. Our aim is to describe the epidemiological and clinical characteristics and outcomes of adult IE patients.

\section{Materials And Methods}

This is a retrospective, descriptive record review study that was approved by the Unit of Biomedical Ethics Research Committee of King Abdulaziz University (KAU). Our aim was to describe the epidemiological and clinical characteristics and outcomes of adult IE patients who were admitted to King Abdulaziz University Hospital (KAUH) in Jeddah, Saudi Arabia, in the period from 2003 until 2019. KAUH is one of the biggest centers in the western region of Saudi Arabia, and it has more than 1000 beds.

We included all adult patients (16 years and above) who were admitted with a definitive diagnosis of IE, according to the Duke Criteria, between 2003 and 2019. Patient data were collected from both paper and electronic medical records. Demographic data (age, gender, nationality, and marital status), type of diagnosis, causative agent, biochemical results, echocardiogram findings, the presence of risk factors, patient comorbidities, and type of management were all documented in our datasheet. The data collection process was confidential, saved and carried out in a secured computer, and all data regarding this study will be removed once completing the study.

Statistical analysis was performed using IBM SPSS Statistics (version 21, IBM Corp., Armonk, NY), and patient confidentiality was ensured. Frequencies, means, standard deviations, and others were calculated to describe the results.

\section{Results}

Out of 105 patients diagnosed with IE, 60 patients met our inclusion criteria, therefore, we excluded 45 patients for the following reasons: 35 were pediatric patients, younger than 16 years old, and 10 either did not have enough data or were possible cases according to the Duke's criteria.

Of the 60 patients, 33 were males (55\%) and 27 were females (45\%). The mean patient age was $48.71 \pm 18.3$ 


\section{Cureus}

years, and the Saudi nationality accounted for 18 patients (30\%), while 42 were non-Saudi (70\%) (Table 1 ).

\begin{tabular}{|l|l|}
\hline Feature & No. (\%) \\
\hline Demographic data & \\
\hline Male & $33(55)$ \\
Female & $27(45)$ \\
Saudi & $18(30)$ \\
Non-Saudi & $42(70)$ \\
\hline
\end{tabular}

\section{TABLE 1: Demographic data}

According to the type of affected valve, 52 of the total patients (86.7\%) had native valve involvement, while in $8(13.3 \%)$ prosthetic valves were affected.

The most common comorbidity the patients had was hypertension (HTN), which accounted for 23 patients (38.3\%), followed by end-stage renal disease (ESRD) in 20 (33.3\%), and DM in 17 (28.3\%). Also, 13.3\% of the patients were known to have RHD, and they were not only with prosthetic heart valves but also native valves.

Fifty-five of the total IE patients had blood cultures taken, 42 of which were positive (70\%), and the most frequent organisms isolated were $S$. aureus, 15 patients (25\%), followed by $S$. viridans, 8 (13.3\%), and coagulase-negative $S$. aureus, 4 (6.7\%) (Table 2).

\section{Feature}

Risk factors

Previous IE

Previous cardiac surgery

Prosthetic heart valve

Cardiac implantable device

RHD

ESRD

Previous dental procedure

Previous event of bacteremia or infection

Organisms

S. aureus

S. viridans

Coagulase-negative $S$. aureus

Management

Medical

Surgical

Antibiotics

Vancomycin

Ampicillin

Ceftriaxone
$15(25)$

4 (6.7)

$58(96.7)$

2 (3.3)

$15(25)$

7 (11.7)

No. (\%)

$9(15)$

$11(18.3)$

10 (16.7)

3 (5)

8 (13.3)

20 (33.3)

3 (5)

$30(50)$

7 (11.7) 


\section{Cureus}

Chemotherapy

Blood culture

Positive

Negative

Echocardiography findings

Vegetations

53 (88.3)

MVR

AVR

TVR

Complications

Sepsis

Stroke

Pulmonary emboli

4 (6.7)

AKI

$4(6.7)$

Brain emboli

3 (5)

Multi-organ failure

Septic emboli

Heart failure

Aortic root abscess

1 (1.7)

Septic shock

1 (1.7)

\section{TABLE 2: Patients' characteristics}

This table demonstrates patients' characteristics including, predisposing factors, causative organism, type of management, antibiotics used, blood culture result, echocardiography findings, and complications.

AKI, acute kidney injury; ESRD, end-stage renal disease; IE, infective endocarditis; MVR, mitral valve regurgitation; AVR, aortic valve regurgitation; TVR, tricuspid valve regurgitation; RHD, rheumatic heart disease.

Twenty-one of the total IE patients had C-reactive protein (CRP) laboratory tests done, and 18 of them showed high levels (30\%). Twenty-one of the patients had erythrocyte sedimentation rates (ESR) evaluated, and ESR was high in 19 patients (31.7\%). All the IE patients had white blood cell (WBC) counts tested: 35 showed leukocytosis (58.3\%), 22 had normal counts (36.7\%), and 3 had leukocytopenia (5\%).

Of the total 60 IE patients, 53 (88.3\%) had vegetations. The anatomical locations of vegetations were as follows: mitral valve 28 (46.7\%), aortic valve 17 (28.3\%), tricuspid valve 7 (11.7\%), and pulmonary valve 3 (5\%). Reports of 3 of the 53 patients did not document the locations of vegetations. Only one patient was found to have vegetations on a pacemaker wire. The majority of patients had a solitary vegetation 42 (79.2\%). Echocardiogram for 35 patients (58.3\%) showed valvular destruction. Nine patients (15\%) presented with severe aortic valve regurgitation, and 8 (13.3\%) presented with severe mitral regurgitation. Ejection fraction was found within the normal range in 36 (69.2\%) and was reduced in 8 (15.4\%). Also, ejection fraction at the first follow-up was within the normal range in 12 patients (20\%); however, for 46 patients, there were missing reports. On the other hand, 29 (48.3\%) presented with complications, and sepsis was found in $16(26.7 \%)$, followed by stroke, pulmonary emboli, and AKI by (6.7\%) for each. The mortality rate was $22(36.7 \%)$.

\section{Discussion}

In this review of cases over 17 years, we found 60 cases that met our inclusion criteria, with a mean age of 48.71 years, and a male to female ratio of 1.2:1. Nashmi and Memish had conducted a local study in Riyadh and found a total incidence of 47 patients diagnosed with IE over 10 years. Their mean age was 38 years, with a 1.6:1 male to female ratio [13]. 
Regarding IE risk factors, we found that 33.3\% of the patients had ESRD, $18.3 \%$ had previous cardiac surgery, $16.7 \%$ had prosthetic heart valves, $15 \%$ had a history of previous IE, $13.3 \%$ had a positive history of RHD, and $5 \%$ had previous dental procedures. Simsek-Yavuz et al. had observed that prosthetic valves and RHD were the two most frequent risk factors, with percentages of $43.5 \%$ and $33.9 \%$, respectively. Moreover, they stated that chronic renal failure and being on dialysis have been associated with increased mortality rates in IE patients [23].

We discovered that 55 of the total IE patients had blood cultures taken, 42 of which were positive (70\%), and the most common organisms isolated were $S$. aureus, 15 (25\%), then $S$. viridians, 8 (13.3\%), and coagulasenegative $S$. aureus, 4 (6.7\%).

These results are compatible with a study that was done in the United States (US) in 2015, which reported that $S$. aureus remains to be the most common cause of IE [20]. This similarity in results can be clarified by the reality that $S$. aureus is the most prevalent cause of all types of IE [26].

On the other hand, there is a previous study, also carried out in the US, from 2018 that found that the most commonly reported organism was S. viridians followed by S. aureus [27].

The conflicting research results may be owing to the modifications in the guidelines that occurred in 2007 [20]. The modifications in the population of patients with IE influenced the trends in the bacterial identity of IE [28].

Several types of research confirmed that the most common site for vegetations was the mitral valve followed by the aortic valve $[22,25]$. Besides that expected result for the location of vegetations, we also found that severe regurgitation comes up slightly more often with aortic than with mitral valve involvement. Alongside the severity of valve destruction, we established that the ejection fraction remains roughly within the normal range, as it does at the next follow-up. On the other hand, a study done in Yemen documented ejection fractions below $40 \%$. Furthermore, they found that the most common complication was heart failure, while in our study, sepsis was the highest occurring complication [29]. It is wise to consider that a variety of our patients seemed to be immunocompromised due to DM and other chronic diseases or medications they were receiving, such as corticosteroids. Unfortunately, mortality was still high in our study with a percentage of $36.7 \%$. A systematic review and a meta-analysis published in 2017 showed the mortality rate in the short term to be $20 \%$, while in the long term, $37 \%$ [30]. Additionally, a study done in Dhahran in 2009 reported a mortality rate of $29.4 \%$ [25]; and research performed in Turkey in 2015 reported a mortality rate of $27.8 \%[23]$.

Regretfully, the explanation behind our high mortality rate could include the high extent of comorbidities our patients had, the high mortality rate of the most frequent causative organism (S. aureus), lack of prompt surgical intervention, and surgical facilities (when they are indicated) because our cardiac surgery center was established in 2006 while our review included patients from as far back as 2002. In this sense, our paper could have some degree of referral bias. Also, IE is still quite uncommon among citizens and residents in Saudi Arabia.

Sepsis was the trending complication in our data, followed by stroke and pulmonary emboli. On the other hand, Heiro et al. demonstrated that neurological complications were the most frequent, proceeded by peripheral emboli [6].

\section{Conclusions}

There was a small number of definitive IE cases over a 17-year span in our institution. Surprisingly, a higher mortality rate was found in our patients and a lower surgical intervention rate in comparison with the literature. Though we acknowledge the limitations of a retrospective chart review study, we can speculate that the high mortality rate may be in part related to higher numbers of virulent organisms, delayed presentation, and lack of prompt surgical intervention.

\section{Additional Information}

\section{Disclosures}

Human subjects: All authors have confirmed that this study did not involve human participants or tissue. Animal subjects: All authors have confirmed that this study did not involve animal subjects or tissue. Conflicts of interest: In compliance with the ICMJE uniform disclosure form, all authors declare the following: Payment/services info: All authors have declared that no financial support was received from any organization for the submitted work. Financial relationships: All authors have declared that they have no financial relationships at present or within the previous three years with any organizations that might have an interest in the submitted work. Other relationships: All authors have declared that there are no other relationships or activities that could appear to have influenced the submitted work. 


\section{References}

1. Holland TL, Baddour LM, Bayer AS, Hoen B, Miro JM, Fowler VG Jr: Infective endocarditis. Nat Rev Dis Primers. 2016, 2:16059. 10.1038/nrdp.2016.59

2. Assiri AS: Clinical and microbiological profiles of infective endocarditis in a tertiary hospital in Aseer region, Saudi Arabia. J Saudi Heart Assoc. 2011, 23:207-11. 10.1016/j.jsha.2011.04.002

3. Geller SA: Infective endocarditis: a history of the development of its understanding . Autops Case Rep. 2013, 3:5-12. 10.4322/acr.2013.033

4. Habib G, Hoen B, Tornos P, et al.: Guidelines on the prevention, diagnosis, and treatment of infective endocarditis (new version 2009): the Task Force on the Prevention, Diagnosis, and Treatment of Infective Endocarditis of the European Society of Cardiology (ESC). Endorsed by the European Society of Clinical Microbiology and Infectious Diseases (ESCMID) and the International Society of Chemotherapy (ISC) for Infection and Cancer. Eur Heart J. 2009, 30:2369-413. 10.1093/eurheartj/ehp285

5. Wallace SM, Walton BI, Kharbanda RK, Hardy R, Wilson AP, Swanton RH: Mortality from infective endocarditis: clinical predictors of outcome. Heart. 2002, 88:53-60. 10.1136/heart.88.1.53

6. Heiro M, Helenius H, Hurme S, Savunen T, Engblom E, Nikoskelainen J, Kotilainen P: Short-term and oneyear outcome of infective endocarditis in adult patients treated in a Finnish teaching hospital during 19802004. BMC Infect Dis. 2007, 7:78. 10.1186/1471-2334-7-78

7. Toyoda N, Chikwe J, Itagaki S, Gelijns AC, Adams DH, Egorova NN: Trends in infective endocarditis in California and New York State, 1998-2013. JAMA. 2017, 317:1652-60. 10.1001/jama.2017.4287

8. Le V, Gill S: Serious complications after infective endocarditis . Dan Med Bull. 2010, 57:A4192.

9. Mocchegiani R, Nataloni M: Complications of infective endocarditis . Cardiovasc Hematol Disord Drug Targets. 2009, 9:240-8. 10.2174/1871529x10909040240

10. Krčméry V, Hricak V, Fischer V, et al.: Etiology, risk factors and outcome of 1003 cases of infective endocarditis from a 33-year National Survey in the Slovak Republic: an increasing proportion of elderly patients. Neuro Endocrinol Lett. 2019, 39:544-9.

11. Pettersson GB, Coselli JS, Pettersson GB, et al.: 2016 The American Association for Thoracic Surgery (AATS) consensus guidelines: surgical treatment of infective endocarditis: executive summary. J Thorac Cardiovasc Surg. 2017, 153:1241-1258.e29. 10.1016/j.jtcvs.2016.09.093

12. Mylotte D, Rushani D, Therrien J, et al.: Incidence, predictors, and mortality of infective endocarditis in adults with congenital heart disease without prosthetic valves. Am J Cardiol. 2017, 120:2278-83. 10.1016/j.amjcard.2017.08.051

13. Nashmi A, Memish ZA: Infective endocarditis at a tertiary care centre in Saudi Arabia: review of 47 cases over 10 years. East Mediterr Health J. 2007, 13:64-71.

14. Marr KA, Sexton DJ, Conlon PJ, Corey GR, Schwab SJ, Kirkland KB: Catheter-related bacteremia and outcome of attempted catheter salvage in patients undergoing hemodialysis. Ann Intern Med. 1997, 127:275-80. 10.7326/0003-4819-127-4-199708150-00003

15. Kamalakannan D, Pai RM, Johnson LB, Gardin JM, Saravolatz LD: Epidemiology and clinical outcomes of infective endocarditis in hemodialysis patients. Ann Thorac Surg. 2007, 83:2081-6. 10.1016/j.athoracsur.2007.02.033

16. Chaudry MS, Carlson N, Gislason GH, et al.: Risk of infective endocarditis in patients with end stage renal disease. Clin J Am Soc Nephrol. 2017, 12:1814-22. 10.2215/CJN.02320317

17. Cahill TJ, Prendergast BD: Infective endocarditis. Lancet. 2016, 387:882-93. 10.1016/S0140-6736(15)000677

18. Ambrosioni J, Hernandez-Meneses M, Téllez A, et al.: The changing epidemiology of infective endocarditis in the twenty-first century. Curr Infect Dis Rep. 2017, 19:21. 10.1007/s11908-017-0574-9

19. Lee PT, Uy FM, Foo JS, Tan JL: Increased incidence of infective endocarditis in patients with ventricular septal defect. Congenit Heart Dis. 2018, 13:1005-11. 10.1111/chd.12667

20. DeSimone DC, Wilson WR, Baddour LM: Trends in infective endocarditis incidence, microbiology, and valve replacement in the United States from 2000 to 2011: the devil is in the details. J Am Coll Cardiol. 2015, 66:1201-2. 10.1016/j.jacc.2015.05.079

21. Bin Abdulhak AA, Baddour LM, Erwin PJ, Hoen B, Chu VH, Mensah GA, Tleyjeh IM: Global and regional burden of infective endocarditis, 1990-2010: a systematic review of the literature. Glob Heart. 2014, 9:13143. 10.1016/j.gheart.2014.01.002

22. Murdoch DR, Corey GR, Hoen B, et al.: Clinical presentation, etiology, and outcome of infective endocarditis in the 21st century: the International Collaboration on Endocarditis-Prospective Cohort Study. Arch Intern Med. 2009, 169:463-73. 10.1001/archinternmed.2008.603

23. Şimșek-Yavuz S, Şensoy A, Kaşıç̧ıŏlu H, et al.: Infective endocarditis in Turkey: aetiology, clinical features, and analysis of risk factors for mortality in 325 cases. Int J Infect Dis. 2015, 30:106-14. 10.1016/j.ijid.2014.11.007

24. Letaief A, Boughzala E, Kaabia N, et al.: Epidemiology of infective endocarditis in Tunisia: a 10-year multicenter retrospective study. Int J Infect Dis. 2007, 11:430-3. 10.1016/j.ijid.2006.10.006

25. Al-Tawfiq JA, Sufi I: Infective endocarditis at a hospital in Saudi Arabia: epidemiology, bacterial pathogens and outcome. Ann Saudi Med. 2009, 29:433-6. 10.4103/0256-4947.57164

26. Brook I: Infective endocarditis caused by anaerobic bacteria . Arch Cardiovasc Dis. 2008, 101:665-76. 10.1016/j.acvd.2008.08.008

27. Yeo I, Kim LK, Park SO, Wong SC: In-hospital infective endocarditis following transcatheter aortic valve replacement: a cross-sectional study of the National Inpatient Sample database in the USA. J Hosp Infect. 2018, 100:444-50. 10.1016/j.jhin.2018.05.014

28. Vincent LL, Otto CM: Infective endocarditis: update on epidemiology, outcomes, and management . Curr Cardiol Rep. 2018, 20:86. 10.1007/s11886-018-1043-2

29. Khaled AA, Al-Noami AY, Al-Ansi M, Faiza AA: Clinical features and outcome of infective endocarditis in yemeni patients treated with empirical antibiotic therapy. Heart Views. 2010, 11:2-9.

30. Abegaz TM, Bhagavathula AS, Gebreyohannes EA, Mekonnen AB, Abebe TB: Short- and long-term outcomes 


\section{Cureus}

in infective endocarditis patients: a systematic review and meta-analysis. BMC Cardiovasc Disord. 2017, 17:291. 10.1186/s12872-017-0729-5 